\title{
CHARACTERIZATION OF LEPTOSPIRA BORGPETERSENII ISOLATES FROM FIELD RATS (RATTUS NORVEGICUS) BY 16S RRNA AND LIPL32 GENE SEQUENCING
}

\author{
Kumaresan Vedhagiri ${ }^{1}$; Kalimuthusamy Natarajaseenivasan ${ }^{1 *}$; Shanmugarajan G. Prabhakaran ${ }^{1}$; Joseph Selvin ${ }^{1}$; \\ Ramasamy Narayanan ${ }^{1}$; Yogesh S. Shouche ${ }^{2}$; Paluru Vijayachari ${ }^{3}$; Sivalingam Ratnam $^{4}$ \\ ${ }^{1}$ Division of Medical Microbiology, Department of Microbiology, School of Life Sciences, Bharathidasan University, \\ Tiruchirappalli - 620 024, Tamilnadu, India; ${ }^{2}$ National Centre for Cell Sciences, Maharastra, Pune - 411 007, India; ${ }^{3}$ Regional \\ Medical Research Centre, Indian Council of Medical Research, WHO Collaborative Centre for Diagnosis, Research, Reference \\ and Training in Leptospirosis, Port Blair - 744 101, Andaman and Nicobar Islands, India; ${ }^{4}$ Tamilnadu Veterinary and Animal
} Sciences University, Chennai - 600 007, India.

Submitted: December 26, 2008; Returned to authors for corrections: July 15, 2009; Approved: August 21, 2009.

\begin{abstract}
The main goal of this study was to evaluate the prevalence of leptospirosis among field rodents of Tiruchirappalli district, Tamil Nadu, India. In total 35 field rats were trapped and tested for seroprevalence by the microscopic agglutination test (MAT). Isolation of leptospires was performed from blood and kidney tissues and characterized to serovar level. Genomospecies identification was carried out using 16S rRNA and lipL32 gene sequencing. The molecular phylogeny was constructed to find out species segregation. Seroprevalence was about $51.4 \%$, and the predominant serovars were Autumnalis, Javanica, Icterohaemorrhagiae and Pomona. Two isolates from the kidneys were identified as serovar Javanica of Serogroup Javanica, and sequence based molecular phylogeny indicated these two isolates were Leptospira borgpetersenii.
\end{abstract}

Key words: Leptospirosis, Leptospira borgpetersenii, lipL32, 16S rRNA.

\section{INTRODUCTION}

Rodents are one of the most common carriers for spreading of Leptospira among all mammals and were first confirmed by isolation in Indian rats (10). Prevalence of leptospirosis in tropical and subtropical countries is common because the countries like India have an ideal niche for the transmission of leptospires. The agriculture fields, especially paddy and grain cultivating fields, are the most favorable places for their survival and procreation. The rodent population is high in these places because they can get their food adequately (15). The rat is the primary carrier for spreading of Leptospira through their excrement especially in the rice fields. Leptospires are spread by the contaminated urine of the infected rodents to the cattle through their grazing fields. This greatly increases the possibilities of spreading leptospires to the rice field workers (1, 14). In earlier days, serological identification involved using the microscopic agglutination test (MAT). This technique was further improved by using specific monoclonal antibodies for serovar level 
identification. Recently, the molecular biology based identification has been introduced for the genomospecies and so far seventeen species have been classified (2). Furthermore, the molecular level identification will be a convenient and simple procedure to perform when compared with the existing techniques. The purpose of the present study is to isolate and characterize the species level of the leptospires of the rodent (Rattus norvegicus) using 16S rRNA and lipL32 gene sequencing. In addition, the molecular phylogenetic analysis were done for the 16S rRNA and lipL32 for the genomospecies level identification.

\section{MATERIALS AND METHODS}

\section{Study area and samples}

The study area is in the rural range of the Tiruchirappalli district located in Tamil Nadu, South India. In the study area, temperature ranges from $28^{\circ} \mathrm{C}$ to $38^{\circ} \mathrm{C}$. and the geographical position of this area is in North latitude between 10 and 11 - 30 and East longitude between 77 - 45' and 78 - 50'. The proposed collection areas were entirely engaged with agricultural activities especially cultivation of paddy, sugarcane and groundnut. These are all productive sources for rodent breeding and many possibilities for the pathogen spreading. The entrapment of the rodents was attained prior to the harvesting phase. Thirty five animals were trapped, the maximum amount of blood collection was attained by direct heart puncture and serum was separated, kept at $-20^{\circ} \mathrm{C}$ until use.

\section{Isolation of leptospires}

The isolation of Leptospira was made from direct inoculation of two drops of blood in $5 \mathrm{~mL}$ of EllinghausenMcCullough-Johnson-Harris semisolid medium (EMJH) (Difco-USA) with the addition of $0.15 \%$ of agarose (Sigma) and $10 \%$ Bovine serum albumin (BSA) (5). The trapped field rats were sacrificed by cervical dislocation and the body surface was wiped with $70 \%$ ethanol and dissected aseptically. A sample from both kidneys were acquired using sterile rat toothed forceps and inoculated into EMJH medium tubes, incubated at room temperature $\left(30^{\circ} \mathrm{C}\right)$ in the dark and examined under dark field illumination with intervals of 10 days to check for the growth of leptospires for at least three months $(13,17)$. When growth was perceived, successive transfer was made in liquid and semisolid media until the growth was sufficiently abundant.

\section{Microscopic agglutination test (MAT)}

MAT was performed on the sera samples collected from the rats using twelve live leptospiral strains as antigens. The strains belong to the serogroups Australis (strain Jez Bratislava), Autumnalis (Akiyami A), Ballum (Mus 127), Bataviae (Swart), Canicola(Hond Utrecht IV), Icterohaemorrha giae (RGA), Grippotyphosa (Moskova V), Hebdomadis (Hebdomadis), Javanica (Poi), Pomona (Pomona), Pyrogenes (Pyrogenes) and Semaranga (Patoc I). MAT was done at doubling dilutions starting from 1 in 20. Positive samples were titrated to their end point. All the strains were maintained in EMJH medium with periodical subculture. Seven days old cultures free from contaminations were utilized for the performance of MAT.

\section{Determination of pathogenic status and virulence}

The virulence nature of the isolates was determined in laboratory animals (5). A group of four 30 days old guinea pigs (Approximately $150 \mathrm{~g}$ ) were inoculated intraperitoneally with $2 \times 10^{8}$ leptospires in a final volume of $1.0 \mathrm{~mL}$. Animals were confined in separate cages and monitored daily for the presence of clinical signs and symptoms. The reisolation was performed from the kidney tissues of the inoculated guinea pigs for confirmation. All animal procedures carried out in this study were approved by the Institutional Animal Ethical Committee (Animal ethics approval No. BDU/ IAEC / 2008/ $10)$.

Confirmation of the pathogenic status was also performed by the in-vitro system at $13^{\circ} \mathrm{C}$ and in the presence of 8 azaguanine (Sigma) with a concentration of $225 \mu \mathrm{g} / \mathrm{mL}(8,9)$. The pathogenic strains Jez Bratislava and Wijinberg and the non-pathogenic strains Patoc I and Veldrat Semaranga were included as controls. The growth was judged by estimating the 
densities of the cultures (14).

\section{Serological characterization}

The identification of the isolate to the serogroup level was carried out first by MAT using a panel of 39 anti-Leptospira rabbit anti sera representative of 25 serogroups, following the standard procedure (3). Subsequently, the isolates were tested with rabbit anti sera raised against 12 reference strains of 12 local circulating serovars. After the determination of the serogroup status of the isolates these were further characterized using mouse monoclonal antibodies (mAbs) of serogroup Javanica. The isolates were also sent to WHO/FAO/ Collaborating Centre for Reference and Research on Leptospirosis, Queensland, Australia for further confirmation.

\section{DNA purification}

Genomic DNA was extracted from seven days old isolates grown at $30^{\circ} \mathrm{C}$ in EMJH medium and harvested by centrifugation. The pellet was washed twice and dissolved in $500 \mu \mathrm{L}$ solution I (10 mM Tris, $10 \mathrm{mM}$ of $\mathrm{MgCl}_{2}, 2 \mathrm{mM}$ of ethylenediaminetetra acetic acid (EDTA), $400 \mathrm{mM} \mathrm{NaCl}$ and $10 \mathrm{mM} \mathrm{KCl}$ in $500 \mathrm{ml}$ of Millipore water). $50 \mu \mathrm{L}$ of lyzozyme $(5 \mathrm{mg} / \mathrm{mL}$ dissolved in solution I) was added and followed by incubation at $37^{\circ} \mathrm{C}$ for 15 mins. Then $50 \mu \mathrm{L}$ of $10 \%$ SDS and 5 $\mu \mathrm{L}$ of Proteinase $\mathrm{K}(10 \mathrm{mg} / \mathrm{mL})$ was added and incubated at $65^{\circ} \mathrm{C}$ for 30 mins. $40 \mu \mathrm{L}$ of $5 \mathrm{M} \mathrm{NaCl}$ and $32 \mu \mathrm{L}$ of $\mathrm{CTAB}$ were added and again incubated at $65^{\circ} \mathrm{C}$ for 30 mins. Then equal volume of chloroform and isoamylalcohol (24:1) were added. The content was vortexed and centrifuged at 10,000 g for 10 mins. The supernatant was mixed with $0.6 \%$ volume of chilled ethanol and kept in $-70^{\circ} \mathrm{C}$ for one hour. Centrifuge the vials at 12,000 $\mathrm{g}$ for $30 \mathrm{mins}$, the pellet was air dried, dissolved in $50 \mu \mathrm{L}$ of Tris-EDTA (TE) buffer and stored at $-20^{\circ} \mathrm{C}$.

\section{PCR and gene sequencing}

The species level identification was carried out by $16 \mathrm{~S}$ rRNA and lipL32 gene amplification using the specific primers as per the earlier reports $(6,20)$. Primers $16 \mathrm{~S}$ rRNA (F)-5'GGC GGC GCG TCT TAA ACA TG-3', 16S rRNA (R)-5'-
GTG CCA GCA GCC GCG GTA A-3' and lipL32-F-5' - TTA CCG CTC GAG GTG CTT TCG GTG GTC TGC-3', lipL32R-5' - TGT TAA CCC GGG TTA CTT AGT CGC GTC AGA3 ' were used. The $16 \mathrm{~S}$ rRNA and lipL32 fragments were amplified using a thermal cycler (Eppendorf, Germany). Briefly each $50 \mu \mathrm{L}$ PCR reaction mixture contained approximately $50 \eta \mathrm{g}$ of purified DNA, $0.1 \mu \mathrm{M}$ primers, 250 $\mu \mathrm{M}$ of each dNTPs (Fermentas), $3 \mathrm{mM} \mathrm{MgCl}_{2}, 0.5 \mathrm{U}$ of Taq DNA Polymerase (Fermentas), in $10 \mathrm{mM}$ Tris-HCl (pH 9) and $50 \mathrm{mM} \mathrm{KCl}$. The temperature profile as one cycle at $94^{\circ} \mathrm{C}$ for 3 min, 35 cycles at $94^{\circ} \mathrm{C}$ for $30 \mathrm{~s}, 52^{\circ} \mathrm{C}$ for $30 \mathrm{~s}, 72^{\circ} \mathrm{C}$ for $1.5 \mathrm{~min}$ and a final extension at $72^{\circ} \mathrm{C}$ for $7 \mathrm{~min}$ and lipL32 gene were amplified by initially one cycle of $94^{\circ} \mathrm{C}$ for $5 \mathrm{~min}$ and 35 cycles of $94^{\circ} \mathrm{C}$ for $90 \mathrm{~s}, 51^{\circ} \mathrm{C}$ for $90 \mathrm{~s}, 72^{\circ} \mathrm{C}$ for $2 \mathrm{~min}$ and final extension at $72^{\circ} \mathrm{C}$ for $7 \mathrm{~min}$. Amplified products were evaluated by agarose gel electrophoresis. Before sequencing, the 16S rRNA and lipL32 products were purified by Montage $^{\mathrm{TM}}$ PCR purification kit according to manufacturer instructions (Millipore). The purified products were sequenced using ABI PRISM model 377 automatic sequencer (Applied Biosystems, USA) giving 99 percent sequence accuracy. The sequences were aligned using bioinformatics tools and deposited in the National Center for Biotechnology Information (NCBI), Bethesda, USA.

\section{Phylogenetic analysis}

The 16S rRNA and lipL32 gene sequences of the rat isolates and the highly identical sequences for $16 \mathrm{~S}$ rRNA and lipL32, used in this study were retrieved from NCBI database using Basic Local Alignment Search Tool (BLASTn) and PSIBLAST tool with default parameters. Sequences with more significant identity were aligned with ClustalW algorithm implemented in Molecular Evolutionary Genetics Analysis (MEGA 4.0) using Smith-Waterman substitution matrix and trimmed to consensus and Unweighted Pair Group Method with Arithmetic Mean (UPGMA) trees were constructed with 1000 bootstraps at uniform divergence rates with Jukes and Cantor evolutionary model and 0.25 gamma distribution factors (19). Gaps in the aligned sequences were replaced by Ns in 
BioEdit 7.0.4.1 (7). Posterior probability and conserved regions among closely related sequences were carried by MEGA 4.0 and BioEdit 7.0.4.1.

\section{RESULTS}

The seroprevalence of the present study revealed that, serovar Autumnalis was the predominant serogroup followed by Javanica, Icterohaemorrhagiae and Pomona. The percentage seroprevalence observed was $25.7 \%$ for Autumnalis, $14.3 \%$ for Javanica, $5.7 \%$ each for Icterohaemorrhagiae and Pomona (Table 1). In a total of 35 field rats utilized for this study two leptospiral isolates were recovered from rat kidneys. The isolates showed typical morphology and characteristic motility of the genus Leptospira under dark-field microscopic examination. These strains grew well in aerobic conditions both in EMJH semisolid and liquid medium. They failed to grow in tripticase soy broth in which Leptonema grow well. The optimum temperature for their growth $28-30^{\circ} \mathrm{C}$ and the optimum $\mathrm{pH}$ 7.2-7.4 were recorded. The cells were difficult to stain by the Gram's method but stained well by silver impregnation techniques such as Fontana's method. The isolates were coded as R1R and R1L, which showed moderate growth after 20 days of incubation period. The isolates were inoculated into guinea pigs for the determination of pathogenicity and virulence, the inoculated guinea pigs were died after 12-14 days from the date of inoculation and they showed the clinical symptoms like dullness, fever and rough coat before their death. Further, pathogenicity was confirmed by growth at $13^{\circ} \mathrm{C}$ and in the presence of 8 -azaguanine. The saprophytic strains Veldrat, Semaranga and Patoc I reached a maximum density within 21 days at $13^{\circ} \mathrm{C}$ and in the presence of 8-azaguanine within 15 days of incubation. In contrast, the growth of the isolates as well as the pathogenic reference strains Wijnberg and Jez Bratislava were inhibited at $13^{\circ} \mathrm{C}$ and in the presence of 8-azaguanine even after 21 days of incubation indicating the pathogenic nature of the isolates. The re-isolation was successful from the kidney tissues of the guinea pigs in EMJH semisolid medium. These findings indicate the virulence nature of the isolated strains.

Table 1. Percentage of seroprevalence of leptospires among the field rats (Rattus norvegicus).

\begin{tabular}{lcc}
\hline Serovar & $\begin{array}{r}\text { Frequency } \\
\mathbf{1 8 / 3 5}(\mathbf{5 1 . 4 2})\end{array}$ & Highest Titre \\
\hline Australis & $0(0.0)$ & $<20$ \\
Autumnalis & $9(25.7)$ & 640 \\
Ballum & $0(0.0)$ & $<20$ \\
Bataviae & $0(0.0)$ & $<20$ \\
Canicola & $0(0.0)$ & $<20$ \\
Icterohaemorrhagiae & $2(5.7)$ & 160 \\
Grippotyphosa & $0(0.0)$ & $<20$ \\
Hebdomadis & $0(0.0)$ & $<20$ \\
Javanica & $5(14.3)$ & 320 \\
Pomona & $2(5.7)$ & 160 \\
Pyrogenes & $0(0.0)$ & $<20$ \\
Semaranga & $0(0.0)$ & $<20$
\end{tabular}


Serological characterizations of the isolates were performed by agglutination of the isolates with group sera of 25 serovar-specific reference rabbit antisera as a first method and in the second method the HIS raised for the isolates were reacted against the serovar Javanica and the corresponding antigen to determine the heterologous titre (Table 2). The group sera assay showed a MAT titre of 1 in 5120 and 2560 for the isolates R1L and R1R respectively for serovar Javanica and in the second assay it was 5120 for both the isolates. Based on these findings the two isolates were identified as serovar Javanica of serogroup Javanica. They were screened against a panel of mAbs of serovar Javanica for further evaluation and confirmed as serovar Javanica of serogroup Javanica. A titre of 10240, 2560, 1280, 1280 were observed against the mAbs 98$19,98-12,98-8,20-4$ for the isolate R1R and it was observed as 10240, 5120, 1280 for the isolate R1L respectively. These findings were also confirmed with WHO/FAO Reference Laboratory, Brisbane, Australia.

Table 2. MAT results of the hyper immune sera (HIS) of R1R and R1L against the local circulating leptospiral serovar(s)

\begin{tabular}{lcc}
\hline \multirow{2}{*}{ Hyper immune sera } & \multicolumn{2}{c}{ Isolates as antigen (titre) } \\
\cline { 2 - 3 } & R1R & R1L \\
\hline Javanica & 5120 & 5120 \\
R1L & 10240 & 10240 \\
R1R & 10240 & 20480 \\
\hline
\end{tabular}

The genetic characterization for the genomospecies level identification was performed using $16 \mathrm{~S}$ rRNA sequencing of two isolates exhibited $732 \mathrm{bp}$ for R1R and $641 \mathrm{bp}$ for R1L respectively through BLAST alignment (EU159685, EU159686). The alignment score of these two sequences is $\geq$ 200 and the score value is 1123 bits. The identities between the sequences were $99 \%$. The phylogenetic pattern by UPGMA tree is evidenced that, the isolates $\mathrm{R} 1 \mathrm{R}$ and $\mathrm{R} 1 \mathrm{~L}$ uniquely clustered with different serovars of L. borgpetersenii with 100
$\%$ bootstrap confidence value. The closest neighboring clusters include strains of L. biflexa, L. kirschneri and L. interrogans showed $100 \%$ bootstrap confidence values. Based on the phylogenetic analysis, the genetic nature of the isolates confirms that they are members of L. borgpetersenii (Fig. 1). Further the lipL32 gene sequencing of the two isolates exhibited $715 \mathrm{bp}$ for $\mathrm{R} 1 \mathrm{R}$ and $700 \mathrm{bp}$ for R1L respectively through BLAST alignment (EU526389, EU526390). The Blast alignment score was found that $\geq 200$ among the all lipL32 gene sequences, homology was 99 to $100 \%$ and the maximum identity was 97 to $99 \%$. The phylogenetic tree evidenced that these two isolates closely clustered with different serovars like Ballum, Bataviae, and Tarassovi of L. borgpetersenii. The closest neighboring groups include serovars of L. kirschneri and strains L. santarosai clustered $99 \%$ bootstrap confidence value. Even though the lipL32 is highly analogous protein present in all pathogenic Leptospira but the phylogenetic pattern of the present study exhibited the clonality of the sequences of the isolates with the L. borgpetersenii and due to that it may also be used to analyze species segregation (Fig. 2).

Based on the lipL32gene sequence of the isolates, it was further confirmed as belonged to genomospecies L. borgpetersenii.

\section{DISCUSSION}

In South India, paddy is a major source of food and most of the lands are used for its cultivation. It also encourages rodent propagation in the field. The study area Tiruchirappalli is fully surrounded by agricultural fields on the river bank of Cauvery. In the rice cultivation fields the rodent infestation is very common and they are the major reservoir for the dissemination of the leptospires in the surrounding environment (15). Isolation of Leptospira from the rodent population and species level identification by $16 \mathrm{~S}$ rRNA and lipL32 gene sequencing is the first of its kind from Tiruchirappalli. In earlier studies Lahiri reported that significant findings in the urban population of the city of Bombay by isolation of serovar Icterohaemorrhagiae from rats (11). Isolation of serovar Javanica from field rats, laboratory 

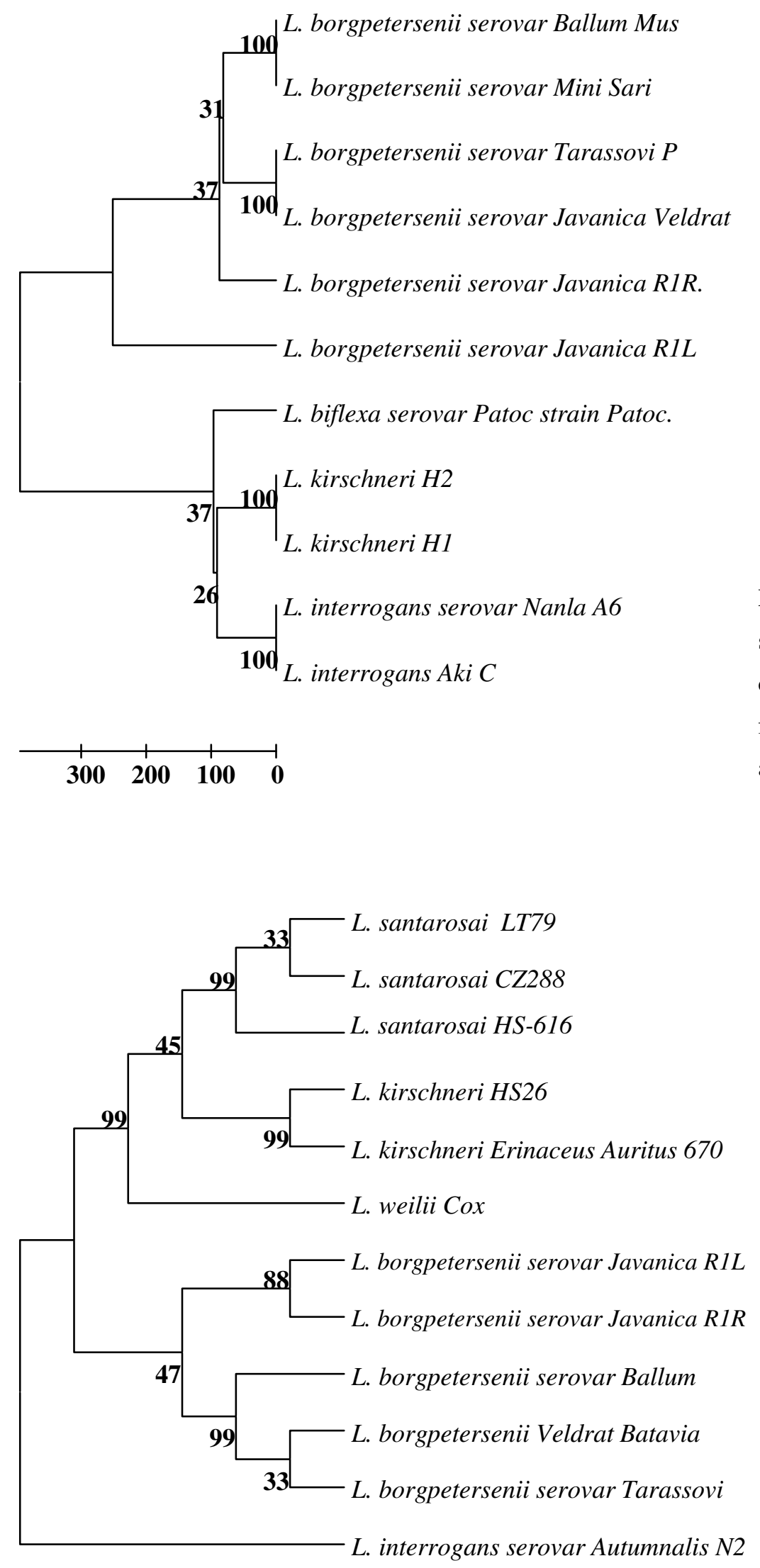

Figure 1. Phylogenetic pattern for $16 \mathrm{~S}$ rRNA sequences of leptospiral isolates (R1R and R1L) in comparison with sequences of representative strains from L. interrogans, L. kirschneri, L. borgpetersenii and L. biflexa.

Figure 2. Phylogenetic pattern for lipL32 gene sequences of leptospiral isolates (R1R and R1L) in comparison with sequences of representative strains from $L$. interrogans, L. kirschneri, $L$. borgpetersenii, L. santarosai and L. weilii. 
mice and wistar rats were done from Chennai and the species level identification of the rat isolates by the gene sequences is a significant contribution to this present investigation (14). The Random Amplified Polymorphic DNA (RAPD) fingerprinting techniques were carried out for the species level identification of the rat isolates of Andaman Islands as L. interrogans, which belonged to serovar Icterohaemorrhagiae of serogroup Icterohaemorrhagiae (16). Even though the RAPD fingerprinting techniques are simple and easy to perform, the consistency and reproducibility is lacking. This may be conquered by the amplification of the specific genes, sequencing and phylogenetic analysis for the clonality to identify the specific species of the leptospiral isolates. Two of the leptospiral isolates $\mathrm{R} 1 \mathrm{R}$ and $\mathrm{R} 1 \mathrm{~L}$ were identified to the serovar level using group sera, followed by monoclonal antibody typing and further they were characterized up to species level using the 16S rRNA and lipL32 gene sequencing. From slaughtered cattle, two leptospiral strains were isolated and identified as Canicola and Copenhageni by monoclonal antibody typing method in Brazil (21). In the present investigation in addition to monoclonal antibody typing the sequence based species identification was also performed. The lipL32 sequences were proved as conserved sequences among the pathogenic leptospires and they were also utilized for the species divergence using the phylogenetic approaches (6). The phylogenetic approach between these isolates showed the clonality, based on the findings the isolates were identified as serovar Javanica of $L$. borgpetersenii. Since the isolation and serological identification of the leptospires seems to be cumbersome and the sequence based molecular identification may make a way for the easy understanding of the species diversification of the leptospires. As a first step this was carried out for the isolates from Tiruchirappalli and it has revealed the existence of $L$. borgpetersenii species in this locality. This study also reported that, the species level identification through molecular tools may explore the diversification of the leptospiral species in various geographical areas to find out prevalence and existence of the leptospires in the clinical samples and environment. While the
Leptospira serovar Javanica has also been isolated from human cases as well as from the rodent population, this reveals the transmission of the leptospires from the rodent carriers as per the present investigation (18). Obviously this approach also leads for the establishment of the molecular based transmission dynamics. However the L. borgpetersenii serovar Javanica is a pathogenic strain, the pathogenicity and the virulence of the strains could also be demonstrated during the study. A previous study carried out in Brazil reported that L. noguchii strain Caco as a sheep isolate and they identified using the 16S rRNA sequencing and the virulence was demonstrated in hamsters (4). In another study two strains of serogroup Grippotyphosa were isolated and identified (12). The rodents are the major contributory source for the spreading of leptospires, the isolation of $L$. borgpetersenii serovar Javanica strain R1R and R1L from Tiruchirappalli give much affordable knowledge for the epidemiology of leptospirosis.

\section{ACKNOWLEDGMENTS}

This study was supported by grants from the Department of Science and Technology (DST), Government of India. The authors thank the Vice-Chancellor, Bharathidasan University for the facilities and thank Dr. L. D. Smythe, WHO/FAO/ Collaborating centre for reference and Research on Leptospirosis, Queensland, Australia for the serovar level confirmation of the leptospiral isolates.

\section{REFERENCES}

1. Bahaman, A.R.; Marshall, R.B.; Blackmore, D.K.; Hathway, S.W. (1980). Isolation of Leptospira interrogans serovar Hardjo from sheep in New Zealand. New Zeal. Vet. J. 28.

2. Bharati, A.R.; Nally, J.E.; Ricaldi, J.N. (2003). Leptospirosis: a zoonotic disease of global importance. Lan. Infect. Dis. 3, 757-771.

3. Dikken, H.; Kmety, E. (1978). Serological typing methods of leptospires. Metho. Microbiol. 11, 260-295.

4. Everton, F.S.; Claudiomar, F.B.; Gustova, M.C.; Bourscheidt, D.; Seyffert, $\quad$ N.; Queiroz, A.; Leiton, $\quad$ S.S.; Ko, A.I.; Dellagostin, O.A. (2007). Isolation of Leptospira noguchii from sheep. Vet. Microbiol. 121, 144-149. 
Vedhagiri, K. et al.

5. Faine, S.; Adler, B.; Bolin, C.; Perolat, P. (1999). Leptospira and leptospirosis. ( $2^{\text {nd }}$ eds), Melbourne, Victoria, Australia. Medical Science p. 1- 259.

6. Haake, D.A.; Chao, G.; Zuerner, R.L.; Barnett, J.K.; Barnett, D.; Mazel, M.; Matsunaga, J.; Levett, P.N.; Bolin, C.A. (2000). The leptospiral major outer membrane protein LipL32 is a lipoprotein expressed during mammalian infection. Infect. Immun. 68, 2276-285.

7. Hall, T.A. (1999). BioEdit: BioEdit: a user-friendly biological sequence alignment editor and analysis programme for Windows 95/98/NT, Nucl. Aci. Symp. Ser. 41, 95-98.

8. Johnson, R.C.; Harris, V.G.; (1967b). Differentiation of pathogenic and saprophytic Leptospira in growth at low temperature. J. Bacteriol. 94, 27-31

9. Johnson, R.C.; Rogers, P. (1964a). Differentiation of pathogenic and saprophytic Leptospira with 8 - Azaguanine. J. Bacteriol. 88, 1618-623.

10. Knowles, R.; Das Gupta, B.M. (1932). Leptospiral infection in Indian rats. Ind. J. Med. Res. 54, 611-614.

11. Lahiri, M.N. (1941). Studies on Leptospira Icterohaemorrhagiae in rats in Bombay city. Ind. Med. Gaz. 76, 536-538.

12. Lilenbaum, W.; Morais, Z.M.; Gonçales, A.P.; deSouza, G.O.; Richtzenhain, L.; Vasconcellos, S.A. (2007). First isolation of leptospires from dairy goats in Brazil. Braz. J. Microbiol., 38, 507-510.

13. Natarajaseenivasan, K.; Ratnam, S.; Ramadass, P.; Manual, P.S.H. (1996). Persistence of dinger's rings by Leptospira interrogans serovar Australis in semi solid EMJH medium. Ind.Vet. J. 73, 571-572.
14. Natarajaseenivasan, K.; Ratnam. S. (1997). A probable virulence factor of Javanica isolates. Ind. J. Ani. Sci. 68, 139-140.

15. Natarajaseenivasan, K.; Boopalan, M.; Selvanayaki, K.; Suresh, S.R.; Ratnam, S. (2002). Leptospirosis among Rice Mill Workers of Salem, South India. Jap.J. Infect.Dis. 55, 170-173.

16. Natarajaseenivasan, K.; Vijayachari, P.; Sharma, S.; Roy, S.; Sugunan. A.P.; Biswas, D.; Sehgal, S.C. (2005). Phylogenetic relatedness among leptospiral strains belonging to same serovar recovered from patients with different clinical syndromes. Infect. Gent. Evol. 5, 185-191.

17. Ratnam, S.; Subramanian, S.; Adinarayanan, N. (1987). Experimental study with leptospires in Bandicoot Bandicota bengalensis. Ind. J. Experi.Biol. 25, 105-107.

18. Saravanan, R.; Rajendran, P.; Thyagarajan, S.P. (1998). Isolation of Leptospira Javanica from urine sample of an acute renal failure case in Chennai: India. Ind. J. Med. Microbiol. 16, 61- 63.

19. Tamura, K.; Dudley, J.; Nei, M.; Kumar, S. (2007). MEGA 4: Molecular Evolutionary Genetics Analysis (MEGA) Software Version 4.0. Mole. Biol. Evol. 24, 1596- 599.

20. Weisberg, W.G.; Barns, S.M.; Pelleiter, D.A.; Lane, D.J. (1991). 16S ribosomal DNA amplification for phylogenetic study. J. Bacteriol. 173, 697-703.

21. Zacarias, F.G.S.; Vasconcellos, S.A.; Anzai, E.K.; Giraldi, N.; de Freitas, J.C.; Hartskeerl, R. (2008). Isolation of leptospira serovars Canicola and Copenhageni from cattle urine in the state of Parana, Brazil. Braz. J. Microbiol., 39, 744-748. 